\title{
La educación sexual en adolescentes de la comunidad de Tilcuautla, Hgo.
}

M. Belem Lacayo Olivelia

Alina A. Dorantes Rodríguez ${ }^{2}$

Karen Estrada Barraza ${ }^{3}$

Asesor: Margarita Bautista García

\section{Resumen}

El presente trabajo manifiesta la investigación cualitativa de cuáles son las nociones de la educación sexual en 48 adolescentes entre catorce y quince años de edad de la comunidad de Tilcuautla, Hidalgo. El objetivo es indagar la información que les han brindado los profesores, los padres en sus hogares, charlas con amigos, campañas y tratamiento dirigidas a un nivel sociocultural bajo. Se utilizó un tipo de diseño etnográfico realista o mixto porque tiene un sentido parcialmente positivista, ya que se recolectaron datos cualitativos y cuantitativos. Se utilizaron como técnicas de recolección de datos grupos focales de tres sesiones de duración, observación participante y se aplicaron encuestas a tres grupos de adolescentes de segundo y terceros grados de la Telesecundaria 196. Los datos obtenidos se categorizaron para obtener los siguientes resultados: cuentan con información sobre sexualidad y relaciones sexuales; ya no toman en cuenta tabúes sociales y no es frecuente el inicio de la vida sexual en estas edades. Conocen los métodos de anticoncepción y porcentaje de eficacia, pero no son utilizados por falta de recursos. La escuela les ha orientado respecto a la educación sexual, aunque preventivamente y prefieren platicar con sus padres sobre el tema que con sus amigos. Lo que ignoran es acerca de enfermedades de transmisión sexual. Las encuestas arrojaron un $56 \%$ de población que ha recibido una adecuada educación sexual, mientras un $16 \%$ sugiere que es nula y un $58 \%$ no la considera tan relevante o no la tiene.
Descriptores: Educación sexual, aportaciones de la escuela y hogar, enfermedades de transmisión sexual(ETS).

\section{Planateamiento del problema}

En la actualidad, en comunidades rurales no se ha brindado la suficiente información sobre sexualidad de manera oportuna, principalmente a adolescentes que comienzan su vida sexual sin conocer los riesgos y maneras de prevenir daños a su salud como embarazos no deseados o enfermedades de transmisión sexual (ETS). Es posible que en casos en los cuales no se haya dado comienzo a una vida sexual activa, si previamente se da una educación sexual, se conozca más sobre métodos de anticoncepción para evitar futuros inconvenientes respecto a la reproducción (Peña, Vera, Barrera, \& García, 2009).

Dentro del estado de Hidalgo existen algunos datos que son relevantes para esta temática: la juventud es considerada como un desafío para la política actual, puesto que representa el futuro de la economía, el inicio de nuevos núcleos familiares y profesionales; por tanto, es un hecho relevante lo que se refiere a la salud de los mismos y uno de los factores a los que se da prioridad es todo lo que implica la fecundidad, más característico en la población joven que gracias a la Encuesta Nacional de la Dinámica Demográfica (2009 en INEGI, 2012) se conoce que oscila entre los quince y veintinueve años de edad el auge de la reproducción joven. La Encuesta Nacional de la Diná-

1 b_lacayohotmail.com, egresada Lic. Psicología ULSA Pachuca

2 alinaadorantes@yahoo.com.mx, egresada Lic. Psicología ULSA Pachuca

3 karen_estba@hotmail.com, egresada Lic. Psicología ULSA Pachuca 


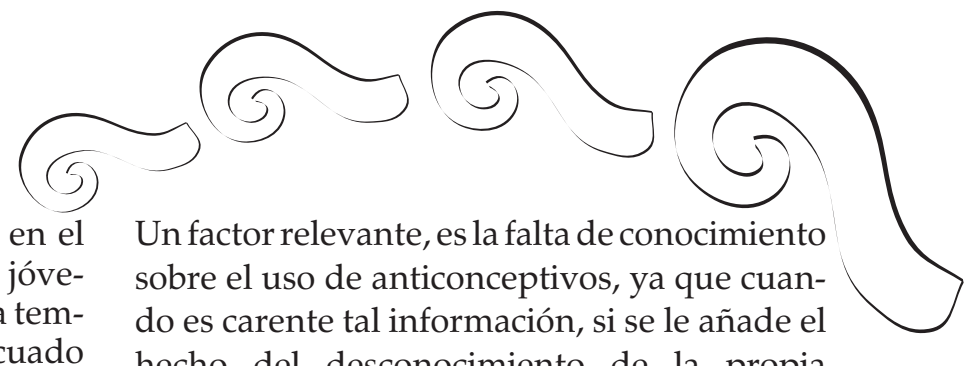

mica Demográfica (ENADID) realizada en el año 2009, muestra la manera en que los jóvenes inician su vida sexual de una manera temprana, sin el previo conocimiento adecuado sobre métodos de anticoncepción, como también sucede con otros métodos de control natal; por ejemplo, en el estado de Hidalgo, un $38.2 \%$ de jóvenes entre 15 y 19 años no utilizaron ninguno en su primera relación sexual, así como un $33.5 \%$ en adultos entre los 20 y 24 años y un $25 \%$ de 25 a 29 años de edad, lo que significa que es un sector que requiere de atención, sobre todo para generaciones posteriores. La ENADID (2009 en INEGI, 2012) también arrojó los resultados sobre el motivo por el que no se usan anticonceptivos: un $19.3 \%$ quiere embarazarse, el $43 \%$ no lo necesita o se encuentran durante el embarazo, $7.4 \%$ tuvo efectos secundarios, del $1.6 \%$ se opone su pareja, 9.3\% está lactando, 3.8\% no conoce la manera para utilizarlos y un $15.6 \%$ presenta respuestas inespecíficas ante la temática para lo que se continúa llevando a cabo programas de información, orientación y toma de decisiones libres respecto a la vida reproductiva joven (INEGI, 2012).

También, ejerce influencia sobre los jóvenes el hecho que se les infunda miedo ante la educación sexual y su revisión constante; pero si se ponen en práctica los programas de salud sexual, incluso puede llegar a reformularse el aspecto de los valores, acuerdos, negociación y decisión, confianza, afecto y seguridad. Para esto, es muy importante tener en cuenta la pertenencia a grupos, la importancia de las escuelas, los padres de familia, y la sociedad que rodea al joven quien habrá de aprender sobre su sexualidad (Peña, Vera, Barrera, \& García, 2009).

La sexualidad implica varios aspectos a tratar como lo son: conocimiento, actitudes, creencias, comportamiento, anatomía, psicología, orientación, funciones de la personalidad, pensamientos e incluso sentimientos. Ejercen influencia los valores éticos, y México es uno de los países más vulnerables ante embarazos no deseados, es por eso que existe también un problema en los jóvenes referente al interés y el compromiso ante las consecuencias de una vida sexual activa (Peña, Vera, Barrera, \& García, 2009).

hecho del desconocimiento de la propia sexualidad, provoca únicamente que aumenten las prácticas sexuales inadecuadas y por consiguiente, infecciones de transmisión sexual (Peña, Vera, Barrera, \& García, 2009).

\section{Objetivos}

El objetivo general de esta investigación es indagar qué entienden por sexualidad los adolescentes de la comunidad de Tilcuautla, Hidalgo, a partir de la educación sexual que han recibido por parte de su familia, de la escuela, y de factores extrínsecos e intrínsecos que ejercen una influencia sobre la percepción de la misma.

Los objetivos específicos se desglosan a continuación:

1. Indagar qué entienden los adolescentes por sexualidad.

2. Conocer qué clase de educación sexual han recibido por parte de su familia.

3. Identificar qué clase de educación sexual han recibido por parte de las escuelas.

4. Conocer la influencia de la religión sobre la percepción de los adolescentes sobre la sexualidad.

5. Averiguar si los adolescentes han recibido educación sexual por parte de un agente externo.

\section{Justificación}

El presente trabajo de investigación se justifica por cuanto se realizó en una población de adolescentes que viven en una comunidad rural, donde es poco considerado para programas de apoyo en esta área. 


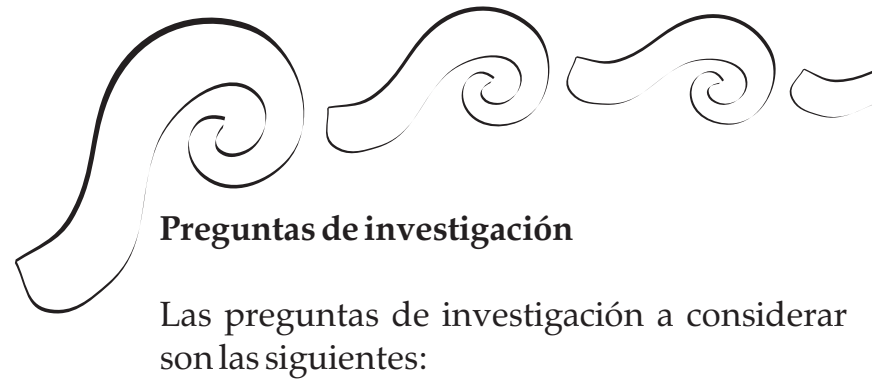

1. ¿Qué entienden los adolescentes por sexualidad?

2. ¿Qué clase de educación sexual han recibido por parte de su familia?

3. ¿Qué clase de educación sexual han recibido por parte de la escuela?

4. ¿Influye la religión en la percepción que tienen los adolescentes sobre la sexualidad?

5. ¿Han recibido los adolescentes educación sexual por parte de un agente externo?

Los adolescentes atraviesan distintas dificultades, como la transición hacia la adultez y hacia la sexualidad, dentro de lo cual se encuentran los cambios puberales que implica una transformación física, emocional y de tipo sexual. Entendemos que la transición hacia la sexualidad adulta comprende algo más que los cambios físicos que se producen en la pubertad, pues el adolescente debe desarrollar nuevos intereses.

Dado a lo anterior, sabemos que los adolescentes se encuentran en una etapa vulnerable debido a estos cambios, donde tomar cierto tipo de decisiones puede cambiar su vida por completo, ya que en cada uno de los adolescentes varía el significado de la sexualidad, el conocimiento del sexo y de los roles sexuales, actitudes hacia el sexo, pautas de conducta sexual, establecimiento de valores en la selección de pareja, etc., y todo esto dependerá, en gran medida, de la educación sexual que han venido recibiendo a lo largo de su vida por parte de la escuela, familia, factores externos, etc.

Es por ello, que en los resultados de este trabajo se pretendió obtener datos de cómo son tomados estos factores por los adolescentes y la relación con el grupo social y cultural al que pertenecen, que en este caso será una comunidad rural, para posteriormente poder tomar algunas medidas y presentar alternativas para mejorar las condiciones en que los adolescentes asumen la sexualidad y desempeñar la función adecuada para miembros de su sexo.

En cuanto a la utilidad metodológica, es de gran importancia ya que se crea una encuesta como instrumento de apoyo por sus implicaciones prácticas, para aplicar a los adolescentes que se pretende estudiar.

Por otro lado, la investigación es viable puesto que la Telesecundaria 196 nos abrió las puertas y es cercana geográficamente a la Universidad La Salle Pachuca. La muestra cubre las necesidades para llevar a cabo la investigación y obtener resultados representativos de dicha comunidad, de las edades en las que es más común que se dé inicio a una vida sexual activa. Se cuenta también con una actitud accesible tanto de investigadoras, como de directivos de la Telesecundaria 196, quienes prestaron su atención y disposición ante la temática a abordar, así como al manejo del tiempo por parte de quienes investigan.

La comunidad de Tilcuautla cubre las características que se requieren para evaluar ciertos aspectos como la influencia de padres, religión, moral, amistades, enfermedades de transmisión sexual, métodos de anticoncepción, entre otros que se toman en cuenta. Asimismo, respecto a recursos y medios tecnológicos se cuenta con cámara fotográfica y video, así como medios de transporte accesibles entre ambas instituciones.

\section{Marco teórico}

La conservación de los derechos y la atención a las necesidades en materia de salud sexual y reproductiva es una condición clave para mejorar la calidad de vida de los adolescentes y una contribución decisiva al desarrollo general de los países. (Ica, 2000) 


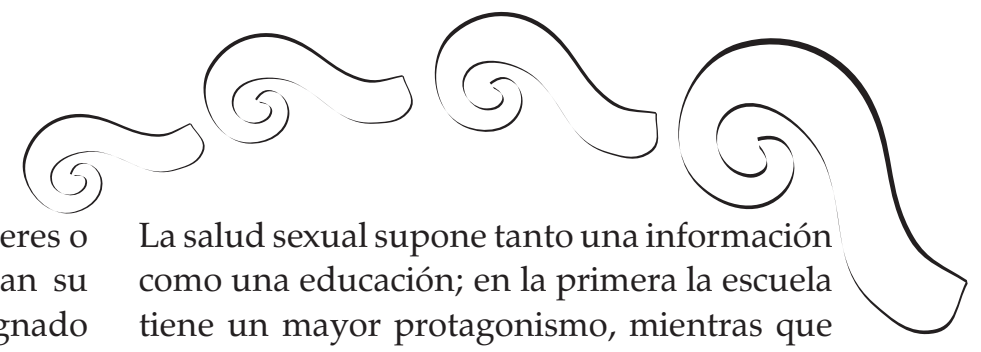

A lo largo de la historia, hombres y mujeres o feminidades y masculinidades, expresan su sexualidad asumiendo un papel asignado socialmente, que estereotipa comportamientos, ahondando las desigualdades de derecho entre ambos. (Carballo, 2002)

Los valores que orientaban los vínculos emocionales entre hombre y mujer establecían relaciones de pareja basados en el dominio, la hostilidad y la incomprensión del hombre sobre la mujer. La mujer y el hombre inmersos en un modelo de familia, de educación, de crianza, de noviazgo y de matrimonio indiscutible, asumían papeles sexuales asignados en perjuicio del desarrollo integral de las personas. (Carballo, 2002)

Por otra parte, Carballo (2002), nos dice que el núcleo familiar, con su estilo particular de vida, es el lugar donde el niño y la niña experimentan el primer proceso de socialización. Lugar donde la acción interpersonal posibilita el "nosotros" a partir del "yo y el "él" y se inicia el aprendizaje de lo que significa ser una persona: hombre o mujer. La información y formación familiares son fuerzas enriquecedoras y transformadoras que permiten el desarrollo de cada ser humano, como único e irrepetible. Asimismo, es en la familia donde primero se empieza a experimentar la expresión de sexualidad por medio del papel genérico asignado. El simple hecho que los padres se comporten como seres sexuados, respetuosos de sí mismos y entre ellos como totalidades, sin desvalorizaciones sexistas, facilita al niño y a la niña el aprendizaje que contribuirá a orientarlo hacia una expresión inteligente de la sexualidad. (Carballo, 2002)

En nuestros días, prácticamente nadie discute la necesidad de introducir en la escuela programas de salud sexual. Es una cuestión en la que deben colaborar intensamente las familias, pues ayuda a los niños y niñas y a los jóvenes a adquirir una educación completa e integral. (Nieda, 1992)

en la segunda, los valores mantenidos por las familias repercuten desde muy temprana edad. De ahí que resulte imprescindible la colaboración entre los padres y la escuela a favor de la coherencia en los planteamientos. (Nieda, 1992)

La educación sexual cumple una función primordial en la formación de las personas: el conocimiento de nosotros mismos, el conocimiento de los demás y las relaciones interpersonales, así como el conocimiento de las instituciones en el contexto social, cultural e histórico en el que vivimos. (López, 2003)

Dentro de comunidades rurales, es relevante conocer algunos aspectos como: si se evita hablar de relaciones sexuales o de enfermedades de transmisión sexual, si al menstruar existe algún tabú o algún hábito de cese de labores de agricultura debido a la misma, si se fomenta la responsabilidad paterna en embarazos precoces, madres solteras, promiscuidad, si se utilizan abortivos con frecuencia, ente otras cosas. (Costa, 2000). Al comparar a las mujeres de las zonas urbanas con las de áreas rurales se puede ver que las últimas tienen mayores posibilidades de empezar una relación sexual antes de los 20 años de edad. Aunque, en las zonas rurales, hay mayor posibilidad de que la primera relación sexual en la adolescencia coincida con el matrimonio. (Departamento Económico y Social, 1996)

Costa (2000) toma en cuenta que tradicionalmente es restringido el hecho de tocar esos temas, ya que se ponen como muy privados, pero lo único que se ha logrado es obstaculizar la cura de enfermedades relacionadas con los órganos sexuales, e interviene la hostilidad e inequidad de género, violencia y coerción a los débiles, baja tolerancia e ignorancia elevada. Para esto, es preciso considerar que las relaciones interpersonales son relevantes y son a su vez, un aspecto que habrá de intentar mejorar- 


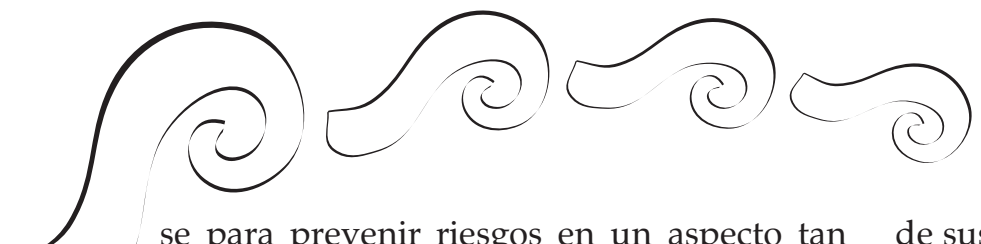

se para prevenir riesgos en un aspecto tan importante como lo es la sexualidad.

Caricote (2007), menciona que la sexualidad es importante para un adolescente para así poder conocer su ser, para poder construirlo, ya que se va reafirmando la identidad; al utilizar la investigación-acción participante, Caricote realiza un estudio en el cual intervienen padres en discusiones grupales, entrevistas abiertas, observación participante, talleres vivenciales, diario de campo, entre otros. Como resultados a tales intervenciones sobre sexualidad, obtuvieron que: se desconoce el tema por parte los padres, con restricciones respecto al placer, con una evaluación negativa por parte de los padres, miedo; usualmente los padres prefieren dejarle estos temas a los docentes cual si fuera su responsabilidad primordial; también predomina la cultura machista y además, toman esto como algo natural o normal; un factor relevante es la desconfianza entre padres e hijos, pues no logran una comunicación adecuada y esto representa que derive de ello el descontrol, el autoritarismo y el temor en los jóvenes hacia los padres.

Ceballos, Arias, y de Bedout (2007), realizaron un estudio en comunidades rurales sobre sexualidad y mencionan otros factores de relevancia que también ejercen fuerte influencia sobre las acciones y la sexualidad de los adolescentes, por ejemplo: alcoholismo, consumo y tráfico de sustancias ilegales, ya que estas situaciones son las que se están dando en bachilleratos públicos dentro de comunidades no desarrolladas.

Indican que los adolescentes del sexo masculino perciben a sus padres como más permisivos que a sus madres respecto a la actividad sexual, pero en ocasiones los padres ni siquiera tienen conocimiento del comportamiento sexual de sus hijos.

Las condiciones socioeconómicas, en el caso de las mujeres, determinan en qué momento de sus vidas dan inicio a las relaciones sexuales, es por eso que se recomienda implementar programas y continuar realizando estudios sobre sexualidad para ubicar principales problemáticas, ver otras causas y riesgos y prestarles una atención adecuada (Ceballos, Arias \& de Bedout, 2007).

\section{Metodología}

Enfoque: es cualitativo, ya que es expansivo y se focaliza paulatinamente en conceptos relevantes de acuerdo con la evolución del estudio. Está fundamentado en la experiencia y la intuición, aplicado a un menor número de casos, que en nuestro estudio, en el cual se detectaron diferentes rubros vinculados con el tema de educación sexual, donde apoyados en datos proporcionados por los participantes se logró consolidar una idea de la comunidad estudiada sobre el tema de educación sexual.

Diseño: es etnográfico, realista o mixto. Se eligió este diseño porque tiene un sentido parcialmente positivista, ya que se recolectan datos tanto cualitativos, como cuantitativos. En la parte cuantitativa, se realizó una encuesta con preguntas basadas en el formato de Likert y la parte cualitativa se realizó a partir de la observación participante, grupo focal y bitácoras de la población en estudio durante dos meses en los horarios donde teníamos tiempo de estar con ellos y a su vez nos permitían estar. Este tipo de diseño considera algunas ideas preconcebidas antes del ingreso al campo y otras no, estas últimas emergieron del trabajo en el campo, lo cual nos permitió establecer las categorías de estudio: percepción de sexualidad, información por agentes externos, información por parte de los padres, anticoncepción, enfermedades de transmisión sexual (ETS), embarazo, aborto, religión, valores, homosexualidad, pornografía y masturbación, prácticas sexuales no comunes, matrimonio y visión. Con base en estas categorías se realizó una encuesta y se clasificó la información obtenida. 


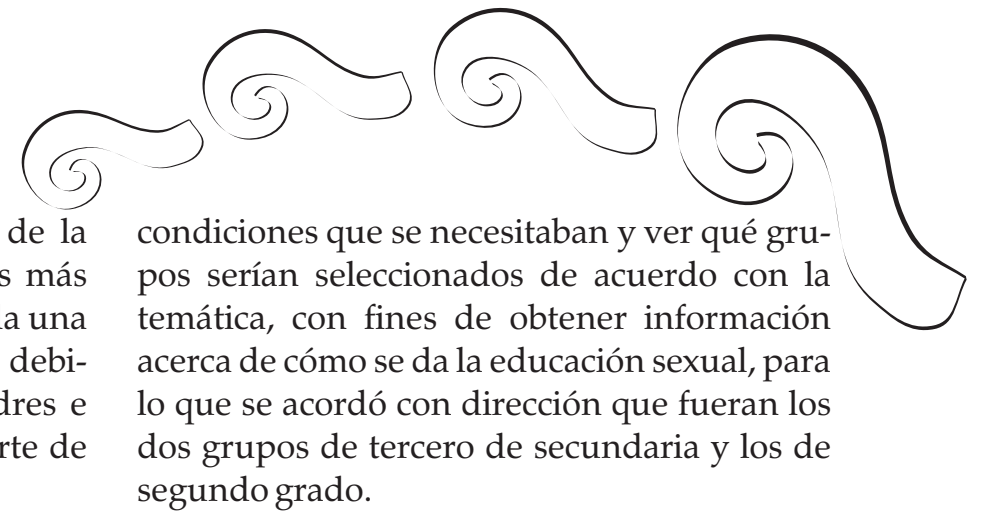

Contexto: se describe como una comunidad rural de escasos recursos en la cual se encuentra la Telesecundaria 196, en la que los alumnos se encontraban en sus respectivas aulas, donde los directivos de la escuela mantienen un control y orden de quien entra y sale, así como de las actividades que se realizan dentro de ésta.

La Telesecundaria se encuentra en un terreno de terracería cercano a la Universidad La Salle Pachuca, es grande y tiene suficientes aulas para grupos numerosos y una cancha donde realizan su clase de deportes; salen los alumnos conforme a sus horarios y no se observaron alumnos fuera de clases.

Población: adolescentes de la comunidad de Tilcuautla, los cuales asisten a la Telesecundaria 196, la cual tiene un grupo de primero, uno de segundo y dos de tercero.

Muestra: es homogénea ya que corresponde a 30 alumnos de los grupos de segundo y los dos terceros de secundaria puesto que es la edad (13 y 14 años) que deseábamos tratar, de sexo indistinto.

Proceso de inmersión al campo de investigación.

\section{Recolección de datos}

Primero, pedimos a la dirección de la Facultad de Ciencias Humanas el justificante por escrito de todo lo que íbamos a realizar, donde se marcaba el objetivo de nuestra investigación. Se llegó a la Telesecundaria 196 de la comunidad de donde se tuvo que pasar a pedir un permiso para realizar la investigación en las

Habíamos decidido realizar historias de vida, entonces como veíamos que los muchachos tenían dificultad para hablar, decidimos realizar un grupo focal, puesto que es la opción de diálogo con mayor cantidad de adolescentes para que se animaran a platicar y no tan estructurado. En este grupo se manifestaron variadas opiniones de los integrantes; aproximadamente de una hora en cada sesión (tres), donde se trataban diversos puntos que refirieron posteriormente las categorías y el cuestionario aplicado de manera más específica. Para ello, se grabaron las sesiones de grupo focal y al escucharlas en análisis nos dimos cuenta que podíamos clasificar las respuestas en categorías que mostraron el interés del grupo y sus problemáticas. Se revisaron las respuestas de los adolescentes que permitieron llegar a conclusiones sobre los conocimientos previos, la manera en que se les educa e informa acerca del tema por parte de padres y profesores, opiniones propias, entre otros aspectos.

Posterior a las grabaciones del grupo focal, pasábamos a agradecer a la dirección por habernos permitido realizar nuestras actividades con los chicos.

Cuando llegábamos de cada sesión con los muchachos acordábamos la hora en que nos veríamos para escuchar las grabaciones de grupo focal y realizar el reporte, así como para comentar lo que habíamos escrito en las bitácoras de todo lo que obteníamos de información al estar conviviendo con los chicos, lo que nos llevaba horas extras de trabajo, ya que era muy interesante lo que cada una de nosotros compartía. 


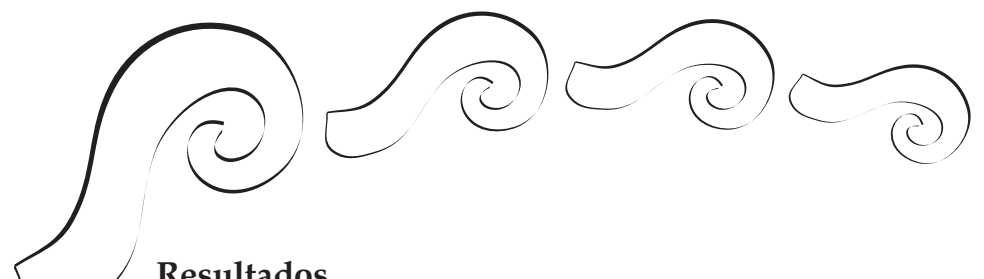

Resultados

Análisis de categorías

A continuación se exponen los datos encontrados en el análisis de los grupos focales a partir de las categorías que se definieron.

\begin{tabular}{|c|c|}
\hline CATEGORÍAS & GRUPO FOCAL \\
\hline $\begin{array}{l}\text { Percepción de } \\
\text { Sexualidad }\end{array}$ & $\begin{array}{l}\text { Uno de los integrantes del grupo focal menciona que la sexualidad } \\
\text { está relacionada a los aparatos reproductores. } \\
\text { Otro integrante señala entender la sexualidad por las etapas que se } \\
\text { van atravesando en la vida. }\end{array}$ \\
\hline $\begin{array}{l}\text { Información por } \\
\text { agentes externos }\end{array}$ & $\begin{array}{l}\text { Uno de los integrantes del grupo focal menciona que han recibido } \\
\text { información sexual por parte de otras universidades y les han } \\
\text { hablado sobre prevención y la edad apropiada para comenzar su } \\
\text { vida sexual. } \\
\text { Un integrante comenta que ha recibido información en casa. } \\
\text { Otro integrante dice que ha recibido información por parte del } \\
\text { Centro deSalud dela Comunidad. }\end{array}$ \\
\hline $\begin{array}{l}\text { Información por parte } \\
\text { de los padres }\end{array}$ & $\begin{array}{l}\text { Un integrante señala que sus padres le hablaron de sexualidad } \\
\text { desde los doce años, antes de entrar a secundaria. } \\
\text { Otro Integrante comenta que al ser él de sexo masculino, se siente } \\
\text { más cómodo hablado con su padre sobre temas de sexualidad. } \\
\text { De igual manera, una adolescente dice sentir más confianza } \\
\text { platicando con su madre sobre el tema. } \\
\text { Otro integrante dice recordar que cuando iba en quinto de } \\
\text { primaria, sus padres se sentaron a platicar con él sobre sexualidad. }\end{array}$ \\
\hline Anticoncepción & $\begin{array}{l}\text { Un integrante comenta que casi siempre reciben únicamente } \\
\text { información sobre el condón. } \\
\text { Otra integrante comenta que a pesar de recibir más información } \\
\text { sobre el condón, también conoce las pastillas y el DIU. } \\
\text { Respecto a los porcentajes de seguridad, un integrante comenta } \\
\text { que el condón tiene } 99 \% \text { de eficacia para la prevención de } \\
\text { embarazos yETS. } \\
\text { Los tres integrantes coinciden en que el método anticonceptivo } \\
\text { más apropiado es el condón al prevenir no sólo embarazo, sino } \\
\text { tambiénETS. }\end{array}$ \\
\hline
\end{tabular}




\begin{tabular}{|c|c|}
\hline $\begin{array}{l}\text { Enfermedades de } \\
\text { Transmisión Sexual } \\
\text { (ETS) }\end{array}$ & $\begin{array}{l}\text { El conocimiento sobre las ETS de un integrante del equipo fue el } \\
\text { saber que cualquier persona puede contraer alguna y que el uso del } \\
\text { condón es la manera menos probable de contraer alguna al tener } \\
\text { relaciones sexuales. } \\
\text { Otro integrante menciona tener el conocimiento de que hay } \\
\text { enfermedades que sólo transmite el hombre pero en ese momento } \\
\text { nolas recuerda. }\end{array}$ \\
\hline Embarazo & $\begin{array}{l}\text { Todos los integrantes del grupo focal concuerdan en no sentirse } \\
\text { preparados a la edad que tienen para enfrentar un posible } \\
\text { embarazo. } \\
\text { Un integrante resalta la importancia de contar con el apoyo de los } \\
\text { padres en caso de un embarazo. }\end{array}$ \\
\hline Aborto & $\begin{array}{l}\text { Ninguno de losintegrantes manifestó estar a favor del aborto. } \\
\text { Uno de ellos menciona que el bebé no tiene la culpa de lo que } \\
\text { sucedió previamente. } \\
\text { Otro integrante menciona que es cruel el practicar un aborto ya que } \\
\text { el bebé que muere es un ser que ya tenía vida. } \\
\text { Al abrirse el tema a las consecuencias del aborto, uno de los } \\
\text { integrantes menciona el riesgo de morir, otro menciona la } \\
\text { posibilidad de quedar sin matriz, y otro marca la posibilidad de ya } \\
\text { no tener hijos en un futuro que se desee hacerlo. }\end{array}$ \\
\hline Religión & $\begin{array}{l}\text { Una integrante menciona que cuando algunas personas saben que } \\
\text { ya se ha iniciado una vida sexual, señalan a esa persona como } \\
\text { pecadora. } \\
\text { Otro integrante menciona que la religión influye al momento de } \\
\text { tener relaciones sexuales a una edad que se considere aceptable. }\end{array}$ \\
\hline Valores & $\begin{array}{l}\text { Un integrante del equipo menciona que la responsabilidad es un } \\
\text { factor muy importante respecto a la sexualidad, para estar } \\
\text { conscientes que no es cualquier cosa. } \\
\text { Otra integrante menciona el antivalor de la discriminación que } \\
\text { sufren las mujeres que han tenido un bebé a corta edad. } \\
\text { De igual manera, otro integrante menciona que a veces pueden no } \\
\text { dar empleo a mujeres embarazadas. } \\
\text { Se menciona que los hombres y mujeres merecen respeto hacia su } \\
\text { sexualidad. }\end{array}$ \\
\hline Homosexualidad & $\begin{array}{l}\text { Los integrantes coinciden en que cada quien tiene derecho a ejercer } \\
\text { su sexualidad de la manera que se desee. }\end{array}$ \\
\hline
\end{tabular}




\begin{tabular}{|l|l|}
\hline $\begin{array}{l}\text { Pornografía y } \\
\text { masturbación } \\
\text { comúcticas sexuales no }\end{array}$ & $\begin{array}{l}\text { Un integrante señala que las personas que recurren a la } \\
\text { masturbación es porque no tienen una relación estable. } \\
\text { Otro integrante menciona que el ver pornografía es algo que a veces } \\
\text { es tomado como moda y que muchos niños de su edad se quieren } \\
\text { sentir grandes por hablar de eso. }\end{array}$ \\
\hline Matrimonios desconocen sobre ellas. \\
\hline Visión & $\begin{array}{l}\text { Un integrante menciona la importancia del respeto ya que se van a } \\
\text { compartir muchas cosas con la otra persona. } \\
\text { Otro integrante menciona que el matrimonio es únicamente para } \\
\text { tener hijos. }\end{array}$ \\
\hline $\begin{array}{l}\text { Un integrante señala que se debe planear la vida sexual, ya que } \\
\text { tiene muchas consecuencias. } \\
\text { Otro integrante menciona que se debe tener algo estable dentro de } \\
\text { la vida sexual, para darle un buen ejemplo a los hijos. }\end{array}$ \\
\hline
\end{tabular}

\section{Estadística descriptiva}

Los resultados derivados del instrumento de evaluación aplicado fueron los siguientes:

Las encuestas arrojaron $28 \%$ de población que ha recibido una adecuada educación sexual, mientras un $16 \%$ sugiere que es nula y un $56 \%$ no la considera tan relevante, o no la tiene.

En un principio, la impresión del lugar fue que no tenía nada que ver con lo que se imaginaba; se vieron jóvenes responsables, ordenados en sus salones, y al momento de platicar con ellos, se vieron posturas muy distintas y en dos casos, dos adolescentes centrados en su realidad, con sentido de responsabilidad hacia sus acciones y decisiones respecto a su sexualidad.

La percepción respecto a los adolescentes de la Telesecundaria 196, fue al momento de aplicar las encuestas, que algunos de los alumnos lo tomaron con morbo; en especial los del segundo grado de secundaria; pero a pesar de eso, mientras respondían y leían las preguntas, su perspectiva era más crítica y objetiva de lo que se esperaba al inicio; durante el grupo focal, se percibió que los adolescentes han recibido información sobre sexualidad, pero no de una manera equiparable en comparación con zonas urbanas.

Respecto a los sentimientos, era algo complicado el hecho de pedir a los alumnos que se retiraran de las clases, pero conforme fue transcurriendo el tiempo y con los alumnos asignados para apoyar la investigación sobre educación sexual, nos sentimos sorprendidas por los resultados, que por lo visto fueron honestos y objetivos; así como las opiniones orales de los integrantes del grupo focal.

Nos llevamos como experiencia el hecho de que al momento de iniciar una investigación, no se debe ir predispuesto ante cómo se supone que responderán o reaccionarán los entrevistados o las personas que se estudien; se debe ir con miras de ser tolerante, por ejemplo, cuando se llegó con uno de los terceros, la profesora hizo que esperara a dar inicio a la aplicación de las encuestas, ya era la hora de salida y los muchachos tenían ganas de irse, pero se tomaron el tiempo de leer y responder de 


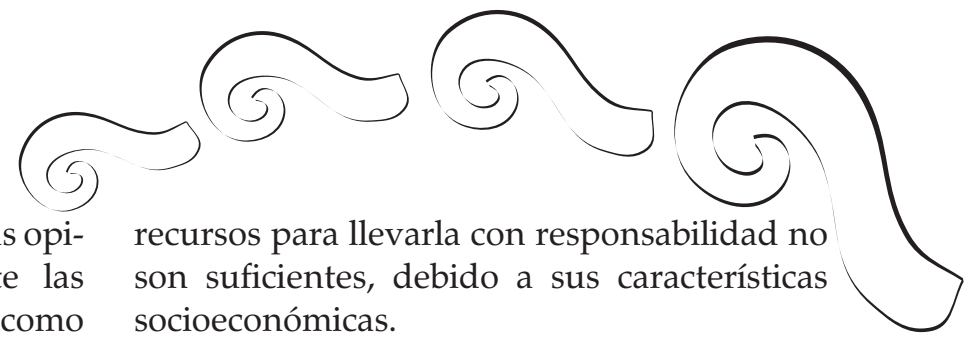

manera honesta, mismo que se vio en sus opiniones cuando dialogábamos durante las grabaciones del grupo focal, en el que como experiencia nos llevamos opiniones que nos parecen muy valiosas, útiles, prácticas, llenas de valores, que no es menos una opinión de un habitante rural, puesto que muchas veces cuentan con más valores y conocimientos que quienes tienen la oportunidad de superarse.

\section{Conclusiones}

En la investigación, se encontró que los adolescentes contaban con información sobre sexualidad; no de manera amplia y detallada, pero tenían conocimientos generales sobre lo que son las relaciones sexuales, algunos de ellos ya no toman en cuenta tabúes sociales, conocen los métodos de anticoncepción, casi todos los que hay y saben su porcentaje de eficacia; aunque en su comunidad, no se utilizan tan frecuentemente debido a la falta de recursos. También, las escuelas les han orientado respecto a la educación sexual, y a diferencia de muchos adolescentes de la ciudad que prefieren platicar del tema con los amigos, la mayoría de los chicos de esta comunidad prefiere platicar con sus padres sobre el tema que con sus amigos, hermanos o profesores.

A diferencia de lo que se creía sobre la educación sexual, se encontró que los jóvenes están conscientes de su sexualidad, que no es tan frecuente el inicio de las relaciones sexuales en esta edad; también consideran varios aspectos para tomar esa decisión, puesto que sí están informados y documentados sobre el tema, aunque en un nivel primario y preventivo, pues a pesar de que hay campañas, no se hizo énfasis en tratamiento en caso de enfermedades de transmisión sexual (ETS), de las que conocen teóricamente a lo que se refieren, así como sus riesgos, pero dentro de las encuestas no les preocupa llegar a padecerlas, ni se registró algún sólo caso. Entonces, los adolescentes de la Telesecundaria 196 de Tilcuautla, Hidalgo, cuentan con información actualizada y suficiente respecto a sexualidad, pero los

Como sugerencia, se tiene el seguir llevando campañas, pero no sólo preventivas, sino de tratamiento para descartar o bien, tratar enfermedades de transmisión sexual (ETS), infecciones y consecuencias que influyan en el pensamiento, sentimientos y conductas. Es decir, tratar los tres niveles de psicología de la salud, orientados a la salud sexual, no únicamente el preventivo, es importante considerar la adherencia a algún tratamiento necesario, así como de las consecuencias que esto conlleva para salud sexual, prevención y control natal, entre otros aspectos.
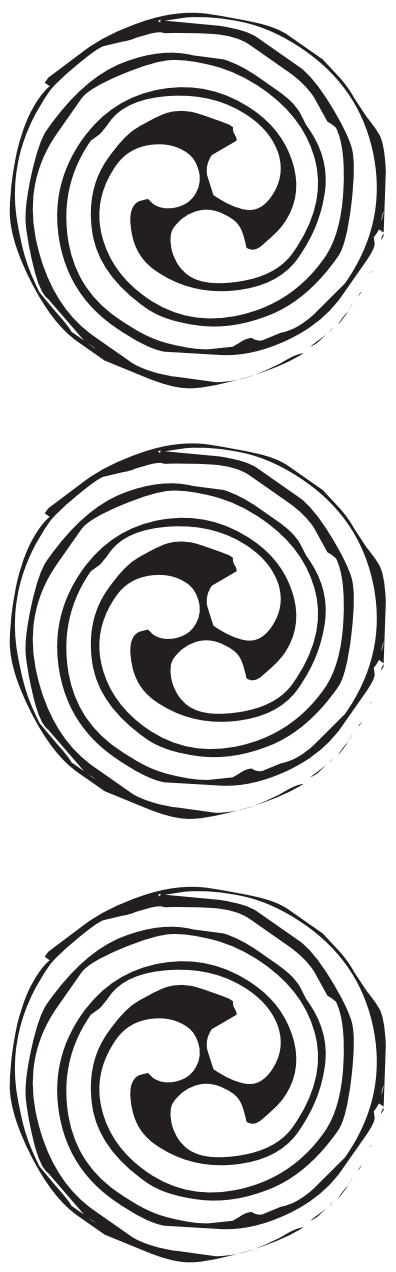


\section{Fuentes de consulta:}

Ceballos Ospino, Guillermo Augusto, De Bedout Hoyos, Andrés, Campo Arias, Adalberto. Relaciones sexuales en estudiantes de secundaria de las zonas rurales del Distrito de Santa Marta. Colombia. Pensamiento Psicológico [en línea] 2007, 3 (julio-diciembre) : [fecha de consulta: 7 de mayo de 2013] Disponible en:<http://www.redalyc.org/ articulo.oa$? \mathrm{id}=80103908>$ ISSN $1657-8961$

Carballo, V., S. (2002) Educación de la expresión de la sexualidad humana. Revista de la Universidad de Costa Rica, 26(1), 31-33. Recuperado de http://redalyc.uaemex.mx/ redalyc/pdf/440/44026104.pdf

De la Concepción, P., O. (2002) Información sexual en un grupo de adolescentes. Revista Cubana de Medicina Militar, 31(4). Recuperado de http://scielo.sld.cu/scielo.php? pid $=$ S013865572002000400006\&script $=$ sci_arttext

Departamento Económico y Social. (1996). FAO. Recuperado el 13 de Marzo de 2013, de http://www.fao.org/docrep/x5633s/x5633s0i.htm

Ica, G. (2000). Encuentro Regional Sobre Juventud Rural Retos y Desafíos para una Agenda Rural en el Nuevo Milenio. México: Kalu Sue.

INEGI. (2012). Estadísticas a propósito del día internacional de la juventud. Pachuca de Soto.

López, N. S. (2003) Curso de educación afectivo-sexual. España: Netbiblo.

Megías, Ignacio; Rodríguez, Elena; Méndez, Susana; Pallarés, Joan. (2005). Jóvenes y Sexo, 4-10.

Navin, C. (2001). Metodología de Educación sexual. Sierra Centro Norte: Abya Yala.

Nieda., J. (1992). Educación para la Salud, Educación Sexual. España: Secretaría de Estado de Educación.

Nuñez, R. M., Hernández, B., García, C., González, D., \& Walker, D. (2003). Embarazo no deseado en adolescentes, y utilización de métodos anticonceptivos posparto. Salud Pública de México, 45 (1), 92.

Soberanes, J. L. (2010). Igualdad, discriminación y tolerancia en México. Revista Mexicana de Derecho Constitucional (22), 1-14.

Peña, M., Vera, J. Á., Barrera, A., \& García, D. (2009). Diagnóstico e Intervención en Educación Sexual para Comunidades Rurales: CIAD. Recuperado el 18 de abril de 2013, de sitio Web Centro de Investigación en Alimentación y Desarrollo: http://www.ciad.mx/ archivos/desarrollo/publicaciones/PUBLICACIONES/Produccion\%20Academica/Cap\%20de \%20Libro/2009/78.pdf

Torres, Germán. Normalizar: discurso, legislación y educación sexual. Iconos. Revista de Ciencias Sociales [en línea] 2009, (Septiembre): [fecha de consulta: 7 de mayo de 2013] Disponible en:<http://www.redalyc.org/articulo.oa?id=50911906003 > ISSN 1390-1249

Vásquez, N. (2007). Cómo hacernos ricos o ahogarnos en el petróleo. Indiana: Author House.
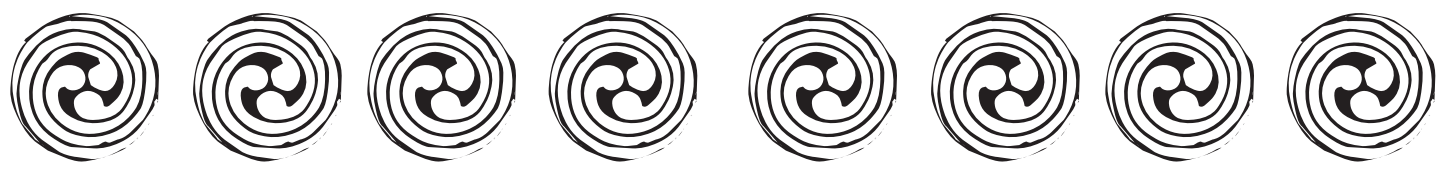

๑ $89 @$ 


\section{ANEXOS}

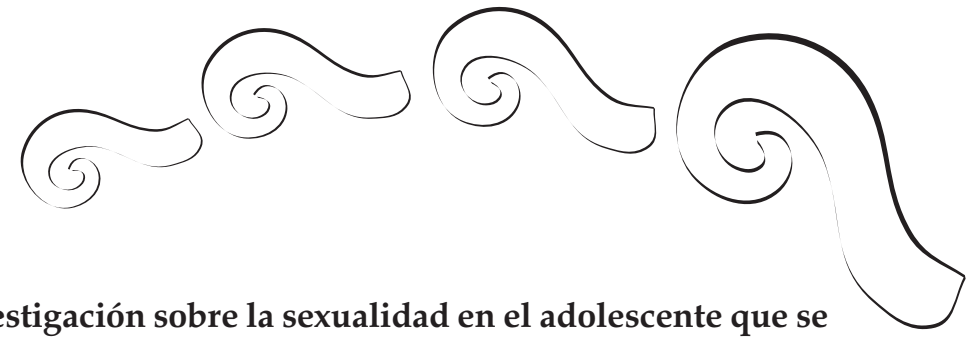

La siguiente encuesta apoyará una investigación sobre la sexualidad en el adolescente que se está realizando en la carrera de Psicología de la Universidad La Salle, por lo que no es necesario que des tu nombre, nos interesa tu honestidad.

1. Me informo constantemente sobre la vida sexual de un chico (a) como yo
a) De acuerdo
c) Indiferente
d) Desacuerdo

2. Mis padres no me hablan de sexo desde que soy pequeño.
a) De acuerdo
c) Indiferente
d) Desacuerdo

3. Tener relaciones sexuales a mi edad es algo malo.
a) De acuerdo
c) Indiferente
d) Desacuerdo

4. He tenido problemas por no saber sobre sexualidad
a) De acuerdo
c) Indiferente
d) Desacuerdo

5. Tengo temor y vergüenza de acudir a un servicio médico para revisión de mis órganos sexuales
a) De acuerdo
c) Indiferente
d) Desacuerdo

6. Me siento incómodo (a) cuando se habla de sexualidad
a) De acuerdo
c) Indiferente
d) Desacuerdo

7. Ya tengo una vida sexual activa
a) De acuerdo
c) Indiferente
d) Desacuerdo

8. Me siento identificado (a) con mi propio sexo
a) De acuerdo
c) Indiferente
d) Desacuerdo

9. Requiero constantemente que mis amigos y/o mi novia (o) demuestren que soy importante para ellos.
a) De acuerdo
c) Indiferente
d) Desacuerdo

10. Necesito que mi novia (o) o la persona con quien tengo relaciones sexuales esté cerca de mí.
a) De acuerdo
c) Indiferente
d) Desacuerdo

11. Me ha costado trabajo tener una relación con una chica (o)
a) De acuerdo
c) Indiferente
d) Desacuerdo

12. Algunos compañeros (as) y/o maestros critican la relación que tengo con mi chica (o)
a) De acuerdo
c) Indiferente
d) Desacuerdo 


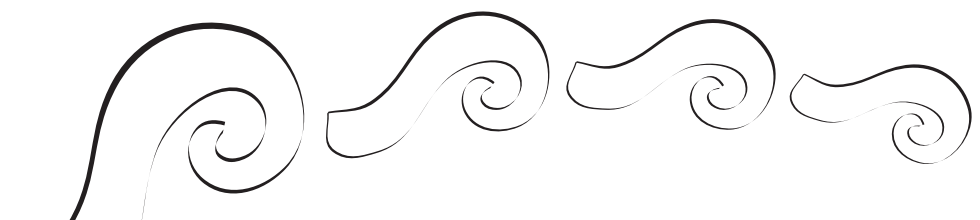

13. Siento culpa porque tengo relaciones sexuales
a) De acuerdo
c) Indiferente
d) Desacuerdo

14. Tengo conflictos constantes con mi novia (o) por causa mía.
a) De acuerdo
c) Indiferente
d) Desacuerdo

15. Permito que mi novia (o) me trate mal
a) De acuerdo
c) Indiferente
d) Desacuerdo

16. Recibo censura constante y me siento frustrado (a) en mi vida sexual
a) De acuerdo
c) Indiferente
d) Desacuerdo

17. Conozco los métodos anticonceptivos y utilizo correctamente alguno para cuidarme
a) De acuerdo
c) Indiferente
d) Desacuerdo

18. La mayor información sobre sexualidad la he recibido de mis amigos (as)
a) De acuerdo
c) Indiferente
d) Desacuerdo

19. He tenido alguna experiencia negativa en mi vida sexual (agresiones, abuso, aborto, embarazo, etc.)
a) De acuerdo
c) Indiferente
d) Desacuerdo

20. Tengo claro que debo llegar virgen al matrimonio
a) De acuerdo
c) Indiferente
d) Desacuerdo

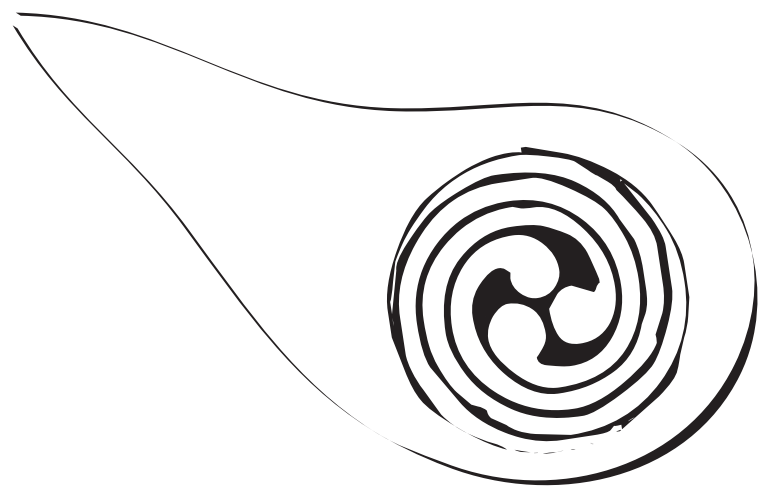



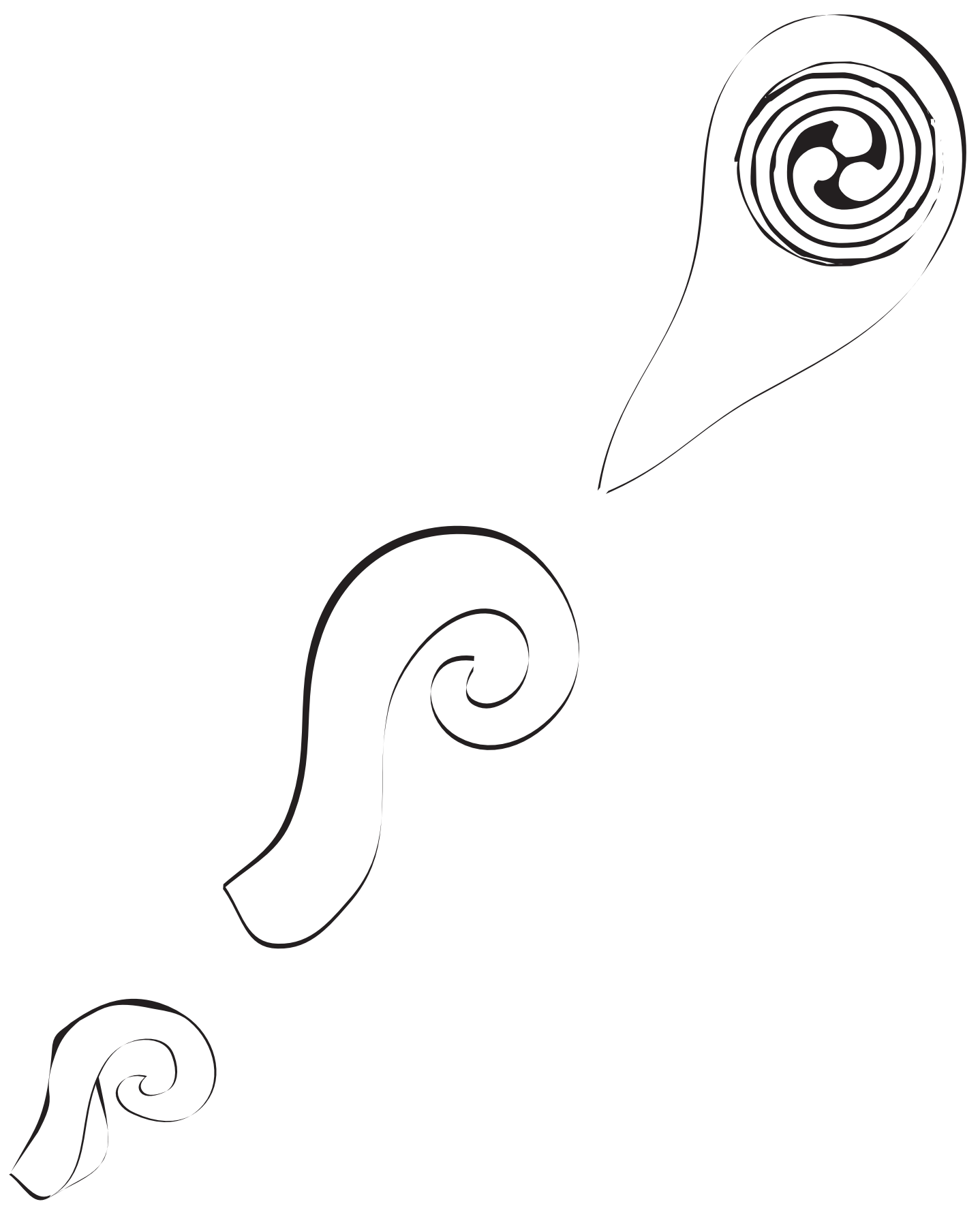

๑92@ 